\title{
miRNA-101-3p.1 as an independent diagnostic biomarker aggravates chronic obstructive pulmonary disease via activation of the EGFR/PI3K/AKT signaling pathway
}

\author{
SHUIFANG CHEN, ZEYING ZHANG, LINA CHEN and JIANLI ZHANG
}

Respiratory Department of Internal Medicine, The First Affiliated Hospital, College of Medicine, Zhejiang University, Hangzhou, Zhejiang 310003, P.R. China

Received January 16, 2019; Accepted June 28, 2019

DOI: $10.3892 / \mathrm{mmr} .2019 .10657$

\begin{abstract}
Exploring independent biomarkers and delineating pathogenic mechanisms could improve the early diagnosis and treatment of chronic obstructive pulmonary disease (COPD). In the present study, a study was conducted to determine the diagnostic potential of miRNA-101-3p.1 in identifying stable COPD (SCOPD) and acute exacerbation of COPD (AECOPD) patients and to reveal the molecular mechanism by which miRNA-101-3p.1 regulates COPD progression. miRNA-101-3p.1 profiles in peripheral blood mononuclear cells of COPD patients were evaluated. Subsequently, receiver operating characteristic curves were created to demonstrate the diagnostic accuracy of miRNA-101-3p.1 in discriminating SCOPD and AECOPD. Finally, the molecular mechanism by which miRNA-101-3p.1 regulates COPD progression was explored. The present study revealed that patients with COPD, and especially patients with AECOPD, had significantly increased levels of miRNA-101-3p.1 and the level of miRNA-101-3p.1 was closely correlated with CAT score and FEV1\% predicted. Notably, miRNA-101-3p.1 accurately discriminated SCOPD and AECOPD. Furthermore, increasing miRNA-101-3p.1 promoted cell proliferation and induced the expression of inflammatory cytokines. Mechanistic investigations revealed that miRNA-101-3p.1 inhibited the expression of von Hippel-Lindau tumor suppressor (pVHL) and ubiquitin conjugating enzyme E2 D1 (UBE2D1). pVHL and UBE2D1 co-upregulated HIF- $1 \alpha$, and HIF- $1 \alpha$ mediated activation of the EGFR/PI3K/AKT signaling pathway. The present results collectively demonstrated that miRNA-101-3p.1 could act
\end{abstract}

Correspondence to: Dr Shuifang Chen, Respiratory Department of Internal Medicine, The First Affiliated Hospital, College of Medicine, Zhejiang University, 79 Qingchun Road, Hangzhou, Zhejiang 310003, P.R. China

E-mail: chen-sf@zju.edu.cn

Key words: chronic obstructive pulmonary disease, diagnosis, miRNA-101-3p.1, ubiquitin conjugating enzyme E2 D1, von Hippel-Lindau tumor suppressor as an independent biomarker for the diagnosis of SCOPD and AECOPD, and that miRNA-101-3p.1 facilitates COPD progression by activating the EGFR/PI3K/AKT signaling pathway.

\section{Introduction}

Chronic obstructive pulmonary disease (COPD) is a chronic disease characterized by persistent airflow restriction and is associated with an abnormal chronic inflammatory response of airways and lung tissues to harmful gases or particles $(1,2)$. Typical clinical manifestations of COPD are chronic cough, sputum, shortness of breath or dyspnea, and wheezing and chest tightness; however, some COPD patients lack clinical manifestations of chronic cough, sputum, or other clinical symptoms before airflow restriction $(3,4)$. Notably, COPD is difficult to precisely diagnose, and it is estimated that only $60-85 \%$ of COPD patients are diagnosed in the early to intermediate stages of disease progression (5). Currently, COPD severity is evaluated primarily according to clinical symptoms, pulmonary function and complications, especially for pulmonary function, it is typically measured by forced expiratory volume in $1 \mathrm{~s} /$ forced vital capacity (FEV1/FVC) after inhalation of a bronchodilator $(6,7)$. Since pulmonary function decreases with age regardless of COPD, using pulmonary function tests to diagnose COPD may cause underdiagnosis in young patients and overdiagnosis in the elderly (8). Thus, timely and accurate COPD is very important for its treatment, and can prevent further destruction of lung tissue.

At present, samples used for COPD diagnosis are derived from lung tissue, sputum, exhaled gas from patients, and bronchial biopsies. Acquiring lung tissue causes trauma and other samples have poor reproducibility and lack standardization $(9,10)$. In recent years, microRNAs (miRNAs) have been revealed to be involved in regulating many physiological and pathological processes including cell differentiation, apoptosis, cell proliferation, and angiogenesis, among others $(11,12)$. Shen et al (13) revealed that smoking induced the downregulation of miR-149-3p, increasing the inflammatory response in COPD patients through the TLR-4/NF- $\mathrm{BB}$ signaling pathway. In addition, 
Liu et al (14) reported that miR-23a was associated with the development of COPD, and identified miR-23a as a potential biomarker to discriminate between frequent and non-frequent exacerbators. miRNA-101-3p.1, a member of the miRNA-101 family, had been identified to interact with COPD-related genes involved in the mechanisms of COPD including imbalance between anti-proteolytic and proteolytic activity, inflammatory response, apoptosis, and oxidative stress (15). Hassan et al (16) revealed that chronic cigarette smoke exposure could induce miR-101 upregulation in the lung of mice and human bronchial epithelial cells, which may suppress CFTR which is involved in the pathogenesis of COPD/emphysema. Notably, miRNA-101-3p.1 was also revealed to be relevant as a non-invasive diagnostic tool to identify acute cellular rejection in heart transplant patients. Thus, it was speculated that miRNA-101-3p.1 may be an ideal diagnostic marker of COPD and may play an important role in the development of COPD.

In the present study, the level, profile, and diagnostic accuracy of miRNA-101-3p.1 were investigated in peripheral blood mononuclear cells (PBMCs) from patients with stable COPD (SCOPD) and acute exacerbation of COPD (AECOPD). Furthermore, the molecular mechanism by which miRNA-101-3p.1 regulates COPD progression was elucidated. This research provides valuable information regarding the accurate diagnosis of COPD.

\section{Patients and methods}

Patients and clinical specimens. All study protocols were approved by The Ethics and Scientific Committees of Zhejiang University. Before study participation, written informed consent was provided by each participant. From October 2015 to September 2017, 58 patients with SCOPD and 46 patients with AECOPD were enrolled in the present study. In addition, 50 age- and sex-matched healthy subjects with normal pulmonary function were also enrolled in this study. The clinical data for all study participants are described in Table I. Based on the Global Initiative for Chronic Obstructive Lung Disease (GOLD) guideline, the criterion for COPD was defined as FEV1/FVC $<0.7$. COPD severity was classified as follows: GOLD I was defined as FEV1 for predicted values (FEV1\%pre) $\geq 80 \%$; GOLD II was defined as $50 \% \leq \mathrm{FEV} 1 \%$ pre $<80 \%$; GOLD III was defined as $30 \% \leq \mathrm{FEV} 1 \%$ pre $<50 \%$; GOLD IV was defined as FEV1\% pre, $<30 \%$. Stable COPD (SCOPD) was identified as a COPD patient that had not undergone acute exacerbation during the last 3 months. Acute exacerbation COPD (AECOPD) patients had a FEV1\%pre 20.41+5.24. AECOPD is defined as an acute worsening of respiratory symptoms such as dyspnea, cough, or sputum purulence severe enough to warrant hospital admission. Frequent exacerbator was identified as AECOPD patients with $2.48 \pm 0.86$ episodes of acute exacerbations during the preceding 1 year. Exclusion criteria included the existence of other chronic lung diseases, nervous system diseases, tumors, diabetes, unstable cardiovascular diseases and liver and kidney diseases. The COPD assessment test (CAT) consists of 8 items with scores ranging from 0 to 5 $(0=$ no impairment). The total scores ranging from 0 to 40 are calculated by adding the score from each item, higher scores indicating a poorer control of COPD or a more severe health status impairment.

Blood samples and PBMC isolation. Venous blood samples of participants were collected in BD $\mathrm{CPT}^{\mathrm{TM}}$ tubes after $12 \mathrm{~h}$ of fasting. The $\mathrm{CPT}^{\mathrm{TM}}$ tubes (BD Biosciences, ) were used to separate PBMCs and plasma from granulocytes and erythrocytes following centrifugation. Subsequently, blood samples were inverted ten times following blood collection and centrifuged at $1,500 \mathrm{x}$ g for $20 \mathrm{~min}$. Then, the PBMC layer was gently suspended in the plasma and transferred to conical tubes and washed with PBS by centrifugation (300 x g; $10 \mathrm{~min})$. Monocyte purity was $>97 \%$ as assessed by flow cytometry. PBMCs were cultured in RPMI-1640 medium with $10 \%$ fetal bovine serum (both from Thermo Fisher Scientific, Inc.) in an incubator at $37^{\circ} \mathrm{C}$ with $5 \% \mathrm{CO}_{2}$.

RNA isolation, reverse transcription, andreversetranscriptionquantitative polymerase chain reaction ( $R T-q P C R)$. Total RNA was extracted for reverse transcription using the PAXgene Blood miRNA Kit (Qiagen, Inc.) following the manufacturer's instructions. Following RNA transcription into cDNA, it was amplified with specific sense and antisense primers using the SYBR Premix Ex Taq II kit (Takara Biotechnology Co., Ltd.) for mRNA and the miRNA PrimeScript RT Enzyme Mix kit (Takara Biotechnology Co., Ltd.) for miRNA. U6 was used as a miRNA internal control; GAPDH was used as a gene internal control. The gene expression level was compared to internal control and calculated using the equation: Fold expression level $=2^{-\Delta \Delta \mathrm{Cq}}$ (17). Independent experiments were performed in triplicate, as were PCR reactions for each gene. The primers were as follows: miRNA-101-3p.1, 5'-CTTCAG TTATCACAGTACTGTA-3'; and U6, 5'-AACGCTTCACGA ATTTGCGT-3'. The primers for pVHL were as follows: Forward primer, 5'-ACATCGTCAGGTCGCTCTAC-3' and reverse primer, 5'-ATCTCCCATCCGTTGATGTG-3'. The primers for UBE2D1 were as follows: Forward primer, 5'-TAG CGCATATCAAGGTGGAGT-3' and reverse primer, 5'-TGG TGACCATTGTGACCTCAG-3'. The primers for GAPDH were as follows: Forward primer, 5'-ACAGTCAGCCGCATC TTCTT-3' and reverse primer, 5'-GACAAGCTTCCCGTT CTCAG-3'.

Transient transfection with siRNAs, miRNA mimics or miRNA inhibitor. siRNA, miRNA mimics and a miRNA inhibitor were designed and synthesized by Sangon Biotech Co., Ltd. PBMCs were plated onto a 6-well plate at 30-50\% confluence. After $24 \mathrm{~h}$, siRNA, miRNA mimics or miRNA inhibitor was transfected into cells via Lipofectamine 2000 (Thermo Fisher Scientific, Inc.) based on the manufacturer's protocol. PBMCs were collected after $48 \mathrm{~h}$ for further experiments. The sequences for the miRNA mimics were: 5'-UACAGUACUGUGAUA ACUGAAG-3' (sense) and 5'-UUCUGUCAUGACACUAUUG ACU-3' (antisense). The sequences of miRNA inhibitor were: 5'-CUUCAGUUAUCACAGUACUGUA-3'. The sequences of the negative controls (NC) were 5'-GUGGAUAUUGUUGAC AUCA-3' (sense) and 5'-dTdTAAGAGGCUUGCACAGUG CA-3' (antisense). The sequences of pVHL siRNA was 5'-CCA AUGGAUUCAUGGAGUA-3' (sense) and 5'-CCACCCAAA UGUGCAGAAA-3' (antisense). The sequences of ubiquitin 
Table I. Clinical characteristics of HS, SCOPD patients and AECOPD patients.

\begin{tabular}{|c|c|c|c|}
\hline Clinical variable & $\mathrm{HS}(\mathrm{n}=50)$ & $\operatorname{SCOPD}(n=58)$ & $\operatorname{AECOPD}(n=46)$ \\
\hline Age, years & $63.47 \pm 11.31$ & $67.53 \pm 8.49$ & $65.13 \pm 9.87$ \\
\hline \multicolumn{4}{|l|}{ Sex } \\
\hline Male $[\mathrm{n},(\%)]$ & $41(82)$ & $49(84.48)$ & $39(84.78)$ \\
\hline Female $[\mathrm{n},(\%)]$ & $9(18)$ & $9(15.52)$ & $7(15.22)$ \\
\hline \multicolumn{4}{|l|}{ GOLD grade } \\
\hline GOLD I & NA & 8 & NA \\
\hline GOLD II & NA & 15 & NA \\
\hline GOLD III & NA & 21 & 24 \\
\hline GOLD IV & NA & 12 & 22 \\
\hline BMI $\left(\mathrm{kg} / \mathrm{m}^{2}\right)$ & $28.64 \pm 4.92$ & $27.65 \pm 4.43$ & $27.11 \pm 4.82$ \\
\hline Duration time (months) & NA & $8.36 \pm 4.11$ & $10.15 \pm 5.26$ \\
\hline Past Smoker $[\mathrm{n},(\%)]$ & $8(16)$ & $49(81.48)^{\mathrm{a}}$ & $42(91.30)^{\mathrm{a}}$ \\
\hline Leukocyte $\left(\mathrm{x} 10^{3} \mu \mathrm{l}\right)$ & $6.47 \pm 2.83$ & $8.03 \pm 2.68^{\mathrm{a}}$ & $8.27 \pm 3.68^{\mathrm{a}}$ \\
\hline Neutrophils $\left(\mathrm{x} 10^{3} \mu 1\right)$ & $3.69 \pm 2.55$ & $5.12 \pm 2.04^{\mathrm{a}}$ & $5.49 \pm 2.81^{\mathrm{a}}$ \\
\hline Lymphocyte $\left(\mathrm{x} 10^{3} \mu 1\right)$ & $2.07 \pm 1.14$ & $2.11 \pm 1.03$ & $1.94 \pm 1.17$ \\
\hline Fibrinogen $(\mathrm{g} / \mathrm{l})$ & $346.17 \pm 153.28$ & $365.81 \pm 115.93$ & $488.36 \pm 193.25^{\mathrm{a}, \mathrm{b}}$ \\
\hline FEV1\% predicted & $93.11 \pm 8.45$ & $54.19 \pm 14.67^{\mathrm{a}}$ & $20.41 \pm 5.24^{\mathrm{a}, \mathrm{b}}$ \\
\hline FVC/FEV1 (\%) & $82.33 \pm 5.36$ & $60.88 \pm 11.10^{\mathrm{a}}$ & $27.92 \pm 9.33^{\mathrm{a}, \mathrm{b}}$ \\
\hline$\beta$-receptor blocker $[\mathrm{n},(\%)]$ & NA & $55(94.83)$ & $45(97.83)$ \\
\hline Steroid drugs $[\mathrm{n},(\%)]$ & NA & $34(58.62)$ & $37(80.43)^{b}$ \\
\hline
\end{tabular}

${ }^{\mathrm{a}} \mathrm{P}<0.01$ vs. the HS group; ${ }^{\mathrm{b}} \mathrm{P}<0.01$ vs. the $\mathrm{SCOPD}$ group. HS, healthy subjects; $\mathrm{SCOPD}$, stable COPD; $\mathrm{AECOPD}$, acute exacerbation of COPD; NA, not available.

conjugating enzyme E2 D1 (UBE2D1) siRNA were 5'-UCU AGCGUCCACAGUGGUTT-3' (sense) and 5'-GCGACAUCU AUGACUCAUTC-3' (antisense). The NC-siRNA sequences were 5'-UUCUCCGAACGUGUCACGUTT-3' (sense) and 5'-AATTCTCCGAACGTGTCACGT-3' (antisense).

Stable expression of $H I F-1 \alpha$. Lentiviral vectors overexpressing $\mathrm{HIF}-1 \alpha(\mathrm{Ov}-\mathrm{HIF}-1 \alpha)$ and siRNA lentiviral vectors inhibiting HIF-1 $\alpha$ expression (Si-HIF-1 $\alpha$ ) were purchased from Cyagen Biosciences. The lentiviral vector expressing scrambled RNA acted as a control (NC). PBMCs were infected with lentiviral vector. Subsequently, flow cytometry sorted fluorescence-activated PBMCs to select polyclonal cells with green fluorescent protein signals. RNA levels from these cell clones was quantified using qRT-PCR. The sequences of siRNA were: 5'-UCA AGUUGCUGGUCAUCAGdTdT-3' (sense) and 5'-CUG AUGACCAGGAACUUGAdTdT-5' (antisense). Primers sequences of HIF-1 $\alpha$ were 5'-TCATCCAAGAAGCCCTAA CGTG-3'(sense); 5'-TTTCGCTTTCTCTGAGCATTCTG-3' (antisense).

Western blot analysis. PBMCs were lysed using RIPA lysate buffer (Beyotime, Institute of Biotechnology) followed by quantification of total protein concentrations using Pierce BCA Protein Assay Kit (Thermo Fisher Scientific, Inc.). Total protein $(50 \mu \mathrm{g})$ was separated using SDS-PAGE gel preparation kit (Beijing Solarbio Science \& Technology Co., Ltd.). After transfer to PVDF membranes (EMD Millipore), 5\% skim milk was used for blocking. Subsequently, the cells were incubated overnight at $4{ }^{\circ} \mathrm{C}$ with a primary antibody and then a secondary antibody was added for $2 \mathrm{~h}$. Signals were detected using the ChemiDoc Touch Imaging System (Bio-Rad Laboratories, Inc.). ImageJ 1.8.0 software (National Institutes of Health) was used to analyze the protein expression level. The primary antibodies and secondary antibody were purchased from Cayman Chemical Company, HuaBio and CST.

3'-UTR luciferase reporter assays. miRNA-101-3p.1 target genes, UBE2D1 and pVHL, were searched using the bioinformatics tool, TargetScanHuman (http:// http://www. targetscan.org). UBE2D1 3'-UTR fragment (2,020 bp) or pVHL 3'-UTR fragment (3,722 bp) was amplified by PCR and cloned into psiCHECK-2 vectors (WT). A GeneTailor Site-Directed Mutagenesis System (Invitrogen; Thermo Fisher Scientific, Inc.) was used for site-directed mutagenesis of miRNA-101-3p.1 (MUT). MT or WT vector and control vector psiCHECK-2 vector were cotransfected into PBMCs with miRNA-101-3p.1 mimics in 48-well plates. Forty-eight hours after transfection, PBMCs were harvested for luciferase assay using Dual-Luciferase Reporter Assay System (Promega Corporation).

Cell proliferation. Cell Counting Kit-8 (CCK-8; MedChemExpress) was used to analyze cell proliferation. PBMCs (1,000-1,500/well) were seeded in 96-well plates and further incubated for $48 \mathrm{~h}$. Next, cell viability was assessed 
A
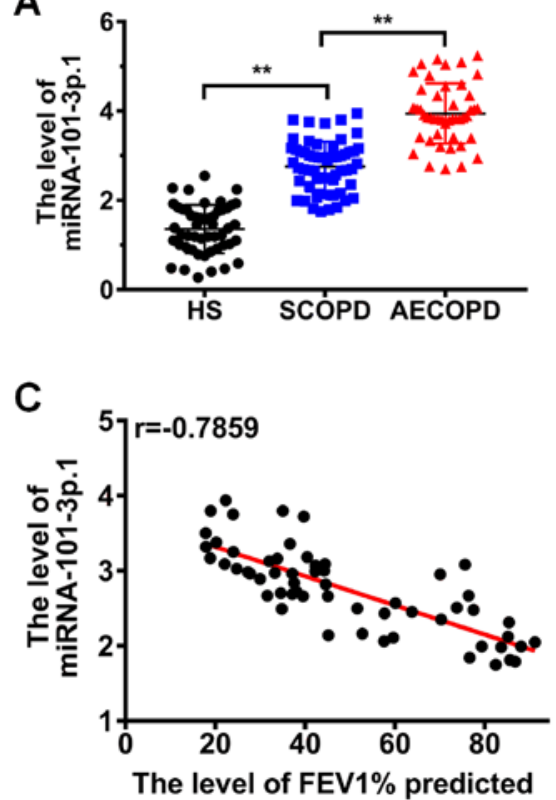

B

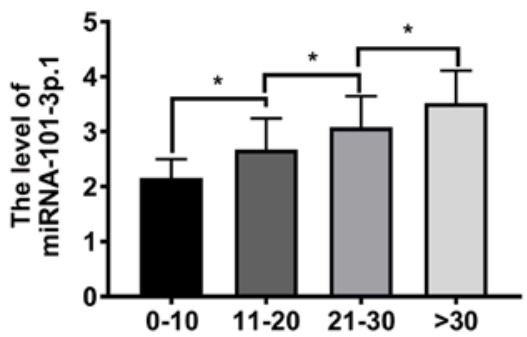

D

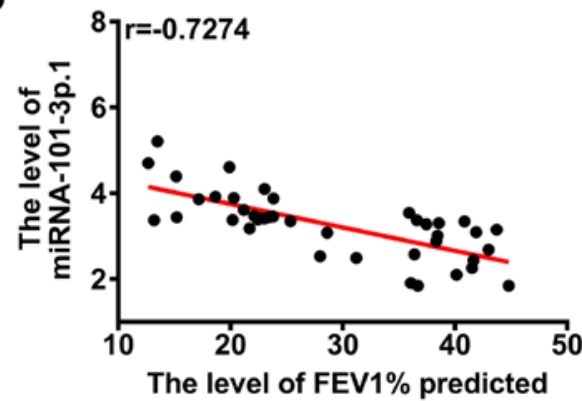

Figure 1. The level profile of miRNA-101-3p.1 in PBMCs. (A) The level of miRNA-101-3p.1 in PBMCs of participants including HS, SCOPD patients and AECOPD patients. (B) The level of miRNA-101-3p.1 of COPD patients with different CAT scores. (C) The relationship between miRNA-101-3p.1 level and FEV1\% predicted in COPD patients based on Pearson correlation analysis ( $\mathrm{n}=58)$. (D) The relationship between miRNA-101-3p.1 level and FEV1\% predicted in AECOPD patients based on Pearson correlation analysis $(\mathrm{n}=46)$. All assays were independently performed in triplicate. $\mathrm{P}<0.05$ and ${ }^{* *} \mathrm{P}<0.01$. $\mathrm{PBMCs}$, peripheral blood mononuclear cells; HS, healthy subjects; COPD, chronic obstructive pulmonary disease; SCOPD, stable COPD; AECOPD, acute exacerbation of COPD.

by adding $100 \mu \mathrm{l}$ medium containing $10 \mu \mathrm{l}$ WST- 8 . The absorbance at a wavelength of $450 \mathrm{~nm}$ was detected using a microplate reader (Tecan Group, Ltd.).

Statistical analysis. All assays were independently performed in triplicate. All data are expressed as the mean \pm standard deviation. Statistical Product and Service Solutions (SPSS) 20.0 software (IBM Corp.) was performed for all statistical analyses. The Chi-square test was used to analyze the count data. The t-test was used for comparisons of data meeting normal distribution between two groups, and those did not conform to normal distribution were compared via non-parametric test. One-way analysis of variance (ANOVA) followed by Tukey's test was applied to compare multiple groups. Receiver operating characteristic (ROC) curves were used to analyze the predictive value and optimal cut-off value of miRNA-101-3p.1. Two-sided P-values $<0.05$ were considered to indicate a statistically significant difference.

\section{Results}

Clinical characteristics of participants. The demographic and clinical characteristics of participants including 58 patients with SCOPD, 46 patients with AECOPD and 50 healthy subjects are presented in Table I. The statistical analyses revealed that there were no significant differences in the distribution of clinical variables including sex, age, and BMI among the three groups and routine blood tests $(\mathrm{P}>0.05)$ except for fibrinogen between the SCOPD group and AECOPD group. The fibrinogen levels in the AECOPD group were significantly higher than that in the SCOPD group and HS group $(\mathrm{P}<0.01)$, but there were no significant differences between the SCOPD group and HS group due to fewer participants. Notably, there were more males than females in the three groups. Among the three groups, there were significant differences in the smoking status. Specifically, the number of past smokers in the SCOPD group and AECOPD group was significantly higher than the healthy subjects $(\mathrm{P}<0.01)$. Notably, FEV1\% predicted and FVC/FEV1 (\%) were significantly decreased in the SCOPD group and AECOPD group relative to healthy subjects $(\mathrm{P}<0.01)$.

The level of miRNA-101-3p.1 in patients with SCOPD or $A E C O P D$. To explore the profiles of miRNA-101-3p.1 in PBMCs, serum samples were collected from healthy subjects, SCOPD patients and AECOPD patients, and total RNA extraction and qRT-PCR were performed. The results presented in Fig. 1A demonstrated that miRNA-101-3p.1 levels in PBMCs were significantly increased in the COPD patients including SCOPD patients and AECOPD patients compared with healthy subjects, and were also significantly higher in AECOPD patients than in SCOPD patients $(\mathrm{P}<0.05)$. To further explore the difference in the level of miRNA-101-3p.1 between the SCOPD patients and AECOPD patients, the relationship between CAT score and the level of miRNA-101-3p.1 was analyzed. The results in Fig. 1B revealed that the miRNA-101-3p.1 levels were significantly increased with the increase of CAT score $(\mathrm{P}<0.05)$. To explore the correlation between miRNA-101-3p.1 levels and COPD progression, a correlation analysis between FEV1\% predicted and miRNA-101-3p.1 level was performed. Pearson correlation analysis, revealed a strong inverse correlation between FEV1\% predicted and the level of miRNA-101-3p.1 in COPD patients $(\mathrm{r}=-0.7859, \mathrm{P}<0.05$; Fig. $1 \mathrm{C})$. In addition, a similar result was observed in AECOPD patients, as revealed 

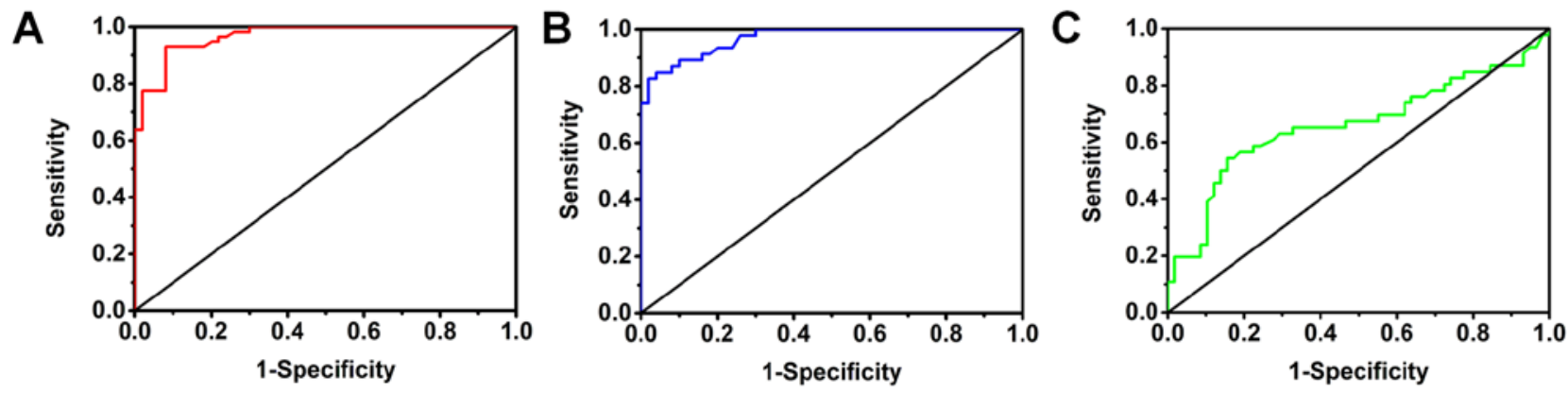

Figure 2. The diagnostic accuracy of miRNA-101-3p.1 in SCOPD and AECOPD was evaluated by ROC curve analyses. (A) A ROC curve was used to discriminate between SCOPD patients and HS. (B) A ROC curve was used to discriminate between AECOPD patients and HS. (C) A ROC curve was used to discriminate between SCOPD patients and AECOPD patients. SCOPD, stable COPD; AECOPD, acute exacerbation of COPD; ROC, receiver operating characteristic; HS, healthy subjects.

A

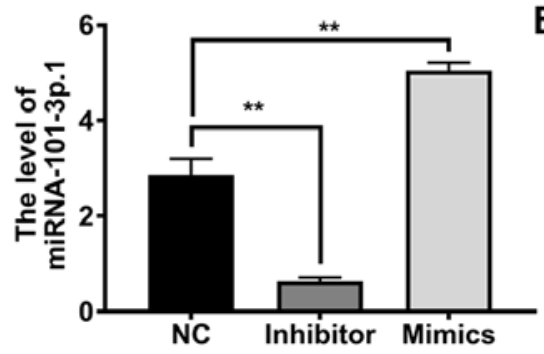

B

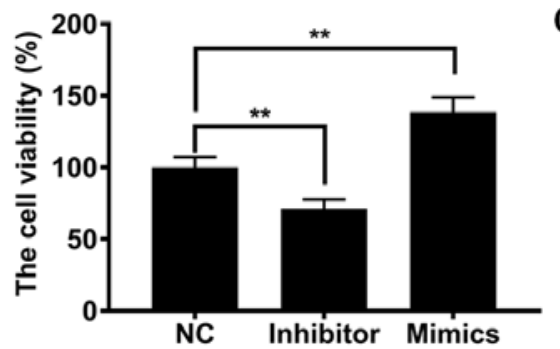

$\mathrm{C}$

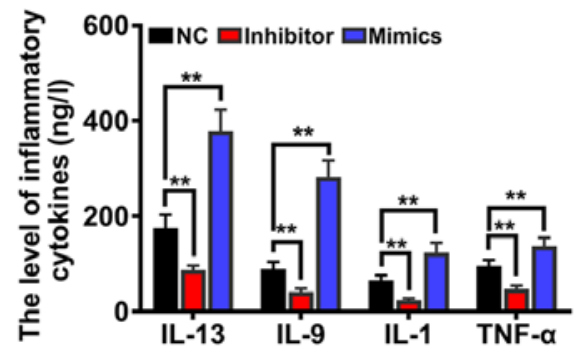

Figure 3. Increasing miRNA-101-3p.1 is responsible for cell viability and the expression of inflammatory cytokines. (A) The miRNA-101-3p.1 levels after transfection of mimics or inhibitor were assessed by RT-qPCR. (B) The effect of miRNA-101-3p.1 on cell viability was evaluated using CCK-8. (C) The effect of miRNA-101-3p.1 on inflammatory cytokines was evaluated by ELISA. All assays were independently performed in triplicate. ${ }^{* *}$ P $<0.01$. RT-qPCR, reverse transcription-quantitative polymerase chain reaction.

in Fig. 1D ( $\mathrm{r}=-0.7274, \mathrm{P}<0.05)$. The aforementioned data collectively indicated that miRNA-101-3p.1 may be used as a diagnostic marker in COPD and may be involved in COPD progression.

Diagnostic accuracy of miRNA-101-3p.1 in SCOPD and $A E C O P D$. The diagnostic accuracy of miRNA-101-3p.1 as an independent biomarker to discriminate SCOPD and AECOPD was evaluated by plotting ROC curves. As is evident in Fig. 2A, miRNA-101-3p.1 could discriminate between SCOPD patients and healthy subjects with AUC values of $0.968(95 \%$ CI: 0.942-0.995; $\mathrm{P}<0.05)$. At the cut-off value of 1.975 for miRNA-101-3p.1, the optimal sensitivity and specificity of miRNA-101-3p.1 were 93.1 and $92.0 \%$, respectively, while Youden's index was 0.851 . Notably, the results presented in Fig. 2B indicated that miRNA-101-3p.1 could also discriminate between AECOPD patients and healthy subjects with AUC values of 0.971 (95\% CI: 0.945-0.997; P<0.05). At the cut-off value of 2.25 for miRNA-101-3p.1, the optimal sensitivity and specificity were 84.8 and $96.0 \%$, respectively, while Youden's index was 0.808 . To further evaluate the diagnostic accuracy of miRNA-101-3p.1 in discriminating between AECOPD and SCOPD, a ROC curve indicated that the AUC value of miRNA-101-3p.1 was 0.661 (95\% CI: 0.549-0.773; P<0.05). At the cut-off value of 3.265 , the optimal sensitivity and specificity were 54.3 and $84.5 \%$, respectively, while Youden's index was 0.388 (Fig. 2C). Collectively, these data indicated that the determination in the level of miRNA-101-3p.1 was effective in detecting SCOPD or AECOPD.
Increasing miRNA-101-3p.1 is responsible for COPD development. To offer sufficient evidence of the role of miRNA-101-3p.1 in COPD diagnosis and progression, miRNA-101-3p.1 mimics, miRNA-101-3p.1 inhibitor and miRNA-101-3p.1 negative control were established (Fig. 3A). Subsequently, the influence on cell proliferation was detected by CCK-8 assay. As revealed in Fig. 3B, compared with NC group, ectopic expression of miRNA-101-3p.1 caused a significant enhancement of proliferation $(\mathrm{P}<0.01)$. However, downregulation of miRNA-101-3p.1 expression resulted in the opposite effect on cell proliferation. Accumulating evidence indicates that inflammatory cytokines play crucial roles in COPD pathogenesis. To evaluate the effects of miRNA-101-3p.1 on pulmonary inflammation responses, inflammatory cytokines including IL-13, IL-9, IL-1 and TNF- $\alpha$ were assessed. These results, presented in Fig. 3C, demonstrated that ectopic expression of miRNA-101-3p.1 significantly enhanced the expression of inflammatory cytokines, including IL-13, IL-9, IL-1 and TNF- $\alpha$, relative to the NC group $(\mathrm{P}<0.01)$. Conversely, downregulating miRNA-101-3p.1 caused marginal expression of IL-13, IL-9, IL-1 and TNF- $\alpha$. Collectively, these results provide clear evidence that increasing miRNA-101-3p.1 promotes cell proliferation and inflammatory responses.

Increasing miRNA-101-3p.1 causes downregulation of UBE2D1 and pVHL in PBMCs. To delineate the molecular mechanism by which miRNA-101-3p.1 promotes the expression of inflammatory cytokines, influencing COPD progression, miRNA-101-3p.1 target genes were searched using 
A
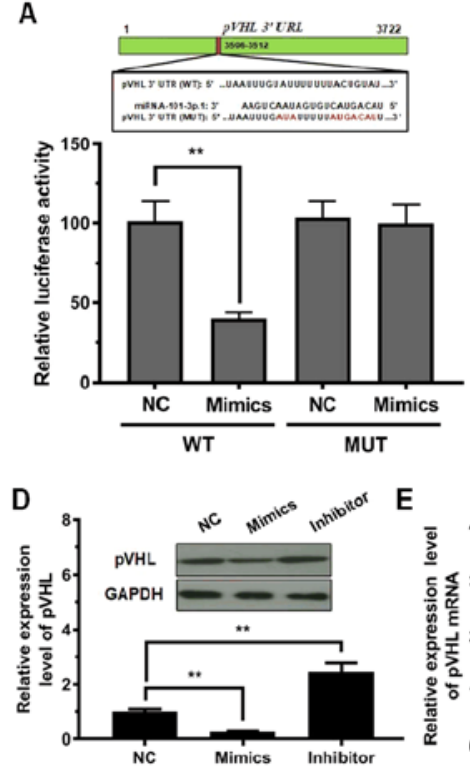

E

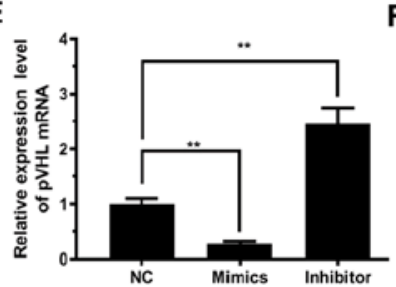

B

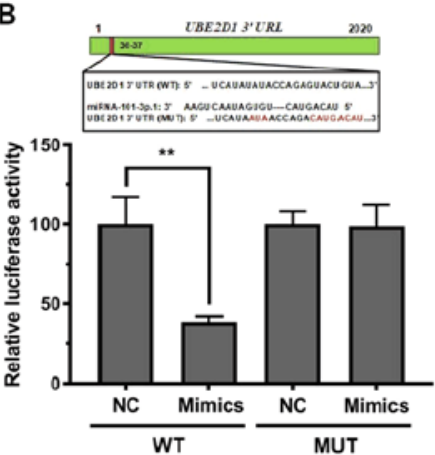

$\mathbf{F}$
C

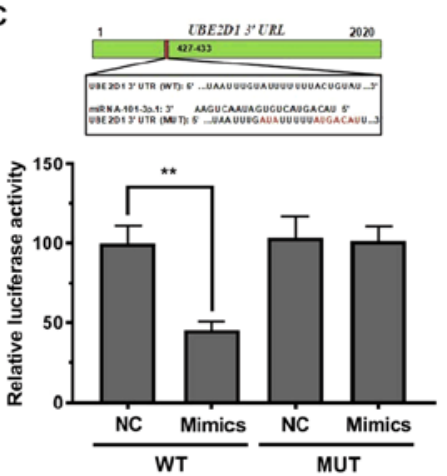

G

Figure 4. miRNA-101-3p.1 directly inhibits the expression of pVHL and UBE2D1 by binding to their 3'-UTRs. (A-C) The effect of miRNA-101-3p.1 on the activity of a luciferase reporter containing either WT or MT 3'-UTR was examined by luciferase reporter gene assays and the sequence alignment between miRNA-101-3p.1 and the 3'-UTR of pVHL and UBE2D1. Western blotting was performed to detect the expression of (D) pVHL and (F) UBE2D1 after transfection with mimics and inhibitor. RT-qPCR was performed to detect the expression of (E) pVHL mRNA and (G) UBE2D1 mRNA after transfection with mimics and inhibitor. All assays were independently performed in triplicate. ${ }^{* *} \mathrm{P}<0.01$. pVHL, von Hippel-Lindau tumor suppressor; UBE2D1, ubiquitin conjugating enzyme E2 D1; RT-qPCR, reverse transcription-quantitative polymerase chain reaction.

the bioinformatics tool, TargetScanHuman (http:// http://www. targetscan.org). This tool predicted 1,100 conserved sites. Among these candidates, pVHL and UBE2D1 are two essential factors involved in cell proliferation, inflammatory reaction, autophagy, and related processes. Thus, a dual-luciferase reporter system was used to verify whether miRNA-101-3p.1 mediated $\mathrm{pVHL}$ and UBE2D1 expression. The 3'-UTR regions of pVHL and UBE2D1 mRNA, including the WT site or MUT site (Fig. 4A-C), were co-transfected with miRNA-101-3p.1 mimics. The results in Fig. 4A indicated that the plasmid with WT pVHL exhibited a significant decrease in luciferase activity after transfection with miRNA-101-3p.1 mimics; however, luciferase activity was not altered after co-transfection with the MUT pVHL and miRNA-101-3p.1 mimics. Similar results were observed with UBE2D1 (Fig. 4B and C).

Subsequently, the expression levels of pVHL mRNA and protein were detected during ectopic expression and silencing of miRNA-101-3p.1. As reported in Fig. 4D and E, in contrast to the NC group, pVHL and UBE2D1 levels were significantly reduced with ectopic expression of miRNA-101-3p.1. Conversely, silencing of miRNA-101-3p.1 resulted in a significant increase in pVHL and UBE2D1 expression. Significantly, the downregulation of pVHL and UBE2D1 was observed with ectopic expression of miRNA-101-3p.1, while upregulation of pVHL and UBE2D1 was observed when downregulating miRNA-101-3p.1 (Fig. 4F and G).

miRNA-101-3p.1 activates the EGFR/PI3K/AKT signaling pathway. To further elucidate the miRNA-101-3p.1-regulated mechanism accounting for the expression of inflammatory cytokines, HIF-1 $\alpha$ expression was investigated, since it is involved in chronic hypoxia. As revealed in Fig. 5A, ectopic expression of miRNA-101-3p.1 significantly increased the level of HIF-1 $\alpha(\mathrm{P}<0.01)$, whereas inhibition of miRNA-101-3p.1 expression had the opposite effect. Next, the decreased expression of pVHL and UBE2D1 was established (Fig. 5B and C). Following the decreased expression of pVHL, the HIF-1 $\alpha$ level was significantly enhanced in PBMCs ( $\mathrm{P}<0.01$, Fig. 5D). Similar results were obtained for UBE2D1, as presented in Fig. 5D. These data indicated that miRNA-101-3p.1 may participate in the regulation of HIF- $1 \alpha$ in inflammatory cytokines. Subsequently, the expression of HIF-1 $\alpha$ was altered by siRNA or overexpression (Fig. 5E). Then the expression of p-PI3K, p-AKT, p-I $\kappa \mathrm{B} \alpha$, EGFR and NF- $\kappa \mathrm{B}$ was assessed by western blotting. As revealed in Fig. 5F, increased HIF-1 $\alpha$ levels were correlated with significantly increased levels of

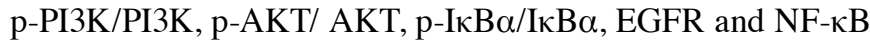
$(\mathrm{P}<0.01)$. The opposite effects were observed on the activation of the EGFR/PI3K/AKT signaling pathway in the absence of HIF-1 $\alpha$. It is concluded from all of these results that increasing miRNA-101-3p.1 aggravates COPD through HIF-1 $\alpha$-dependent activation of the EGFR/PI3K/AKT signaling pathway.

\section{Discussion}

COPD a progressive disease characterized by inflammation and airflow obstruction, is not fully reversible, and is responsible for an increasing number of deaths $(18,19)$. Despite the improved understanding of its pathophysiology, causes of development and management, the molecular mechanisms underlying COPD development are still not fully understood. Effective diagnostic methods for COPD are urgently required. In recent years, dysregulation of miRNAs has been reported to be involved in the development of various pulmonary diseases, including COPD, lung cancer, and emphysema $(20,21)$. Notably, a set of studies reported that miRNAs could affect different 
A

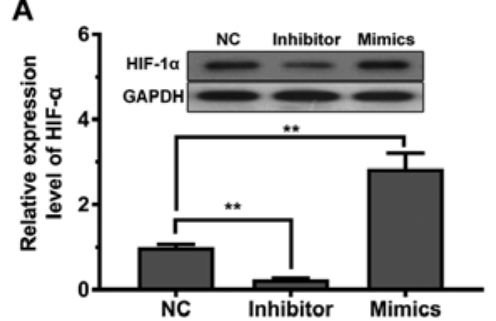

E

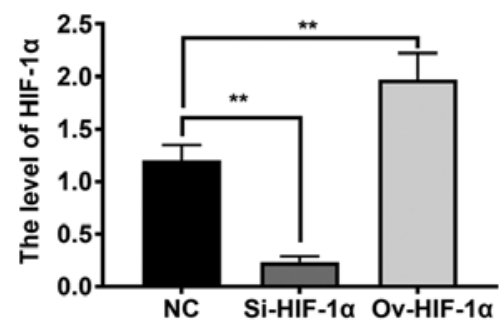

B

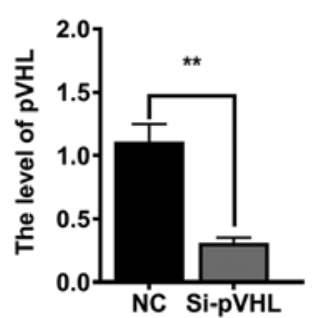

C

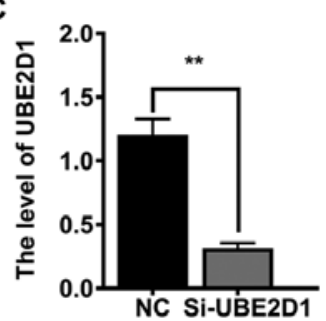

D

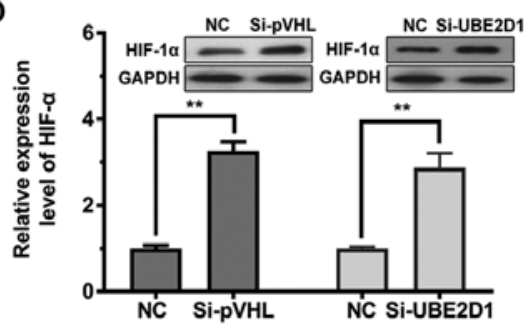

F
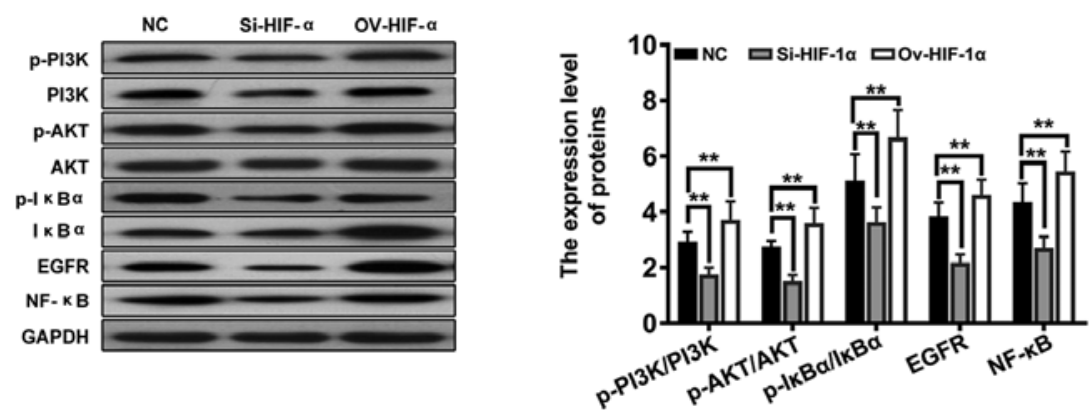

Figure 5. HIF-1 $\alpha$ regulates the EGFR/PI3K/AKT signaling pathway. (A) The effect of miRNA-101-3p.1 on HIF-1 $\alpha$ was detected by western blotting. (B) The pVHL levels after transfection of siRNA were detected by western blotting. (C) The UBE2D1 levels after transfection of siRNA were detected by western blotting. (D) The effect of pVHL and UBE2D1 on HIF-1 $\alpha$ was detected by western blotting. (E) The HIF-1 $\alpha$ levels after knockdown or overexpression of HIF-1 $\alpha$ were detected by western blotting. (F) Western blotting was performed to investigate the effect of HIF-1 $\alpha$ on the EGFR/PI3K/AKT signaling pathway. All assays were independently performed in triplicate. ${ }^{* *} \mathrm{P}<0.01$. pVHL, von Hippel-Lindau tumor suppressor; UBE2D1, ubiquitin conjugating enzyme E2 D1.

biological processes involved in COPD, such as inflammation, tissue repair, and the development of airway lesions $(13,22)$. Investigating miRNAs as contributors to COPD initiation and pathogenesis has potential for advancements in COPD diagnosis. In previous studies, miRNA-101-3p.1 was revealed to be closely related to pulmonary diseases and inflammatory responses $(23,24)$. The present study, was conducted to evaluate the diagnostic potential of miRNA-101-3p.1 to identify SCOPD and AECOPD using PBMCs and to reveal the molecular mechanism by which miRNA-101-3p.1 facilitates COPD progression.

In the present study, it was determined that the miRNA-101-3p.1 level in PBMCs of COPD patients was significantly increased. Especially, the level of miRNA-101-3p.1 in AECOPD patients was significantly higher than that in COPD patients. Further studies revealed that the level of miRNA-101-3p.1 was significantly correlated with the increase of CAT score and the deterioration of pulmonary function. Diagnostically, ROC curves revealed that miRNA-101-3p.1 AUC values were sensitive and specific for discriminating SCOPD and AECOPD. Furthermore, the biological function of miRNA-101-3p.1 was explored and it was revealed that miRNA-101-3p.1 could promote cell proliferation and induce the expression of inflammatory cytokines. Results from target prediction and validation assays indicated that miRNA-101-3p.1 directly inhibited pVHL and UBE2D1 expression by binding to their 3'UTRs. Further studies revealed that pVHL and UBE2D1 co-upregulated HIF-1 $\alpha$, and then promoted activation of the EGFR/PI3K/AKT signaling pathway. Collectively, this provides convincing evidence that miRNA-101-3p.1 could act as a highlight biomarker for the diagnosis of SCOPD and AECOPD, and that miRNA-101-3p.1 facilitates COPD progression by activating the EGFR/PI3K/AKT signaling pathway.
Currently, miRNAs as biomarkers have been widely used for risk assessment, diagnosis, prognosis, and dynamic detection of various diseases, including cancer and cardiovascular disease $(25,26)$. Ramshankar and Krishnamurthy (27) revealed 7 differentially expressed miRNAs by screening lung cancer and COPD patients, demonstrating that miRNAs could be used as biomarkers to differentiate lung cancer and COPD. In the present study, it was revealed that the level of miRNA-101-3p.1 in PBMCs of COPD patients was significantly increased compared with healthy subjects, while the miRNA-101-3p.1 level of AECOPD patients was higher than in SCOPD patients. This observation is consistent with the more serious manifestation of AECOPD. However, Su et al (28) revealed that miRNA-101 was downregulated in hepatocellular carcinoma. Such a discrepancy may be caused by the different contexts in these studies, including tissue types and hepatitis B virus infection. Furthermore, the present research revealed that the miRNA-101-3p.1 level was significantly enhanced with the increase of CAT scores and deterioration of pulmonary function, indicating that the level of miRNA-101-3p.1 may reflect the COPD severity. COPD development may be explained by high levels of miRNA-101-3p.1, leading to progressive pulmonary function damage in COPD patients. Furthermore, these results also indicated that miRNA-101-3p.1 may be an ideal biomarker for the differentiation of SCOPD and AECOPD. Therefore, ROC curve analyses were performed and it was revealed that miRNA-101-3p.1 was able to discriminate SCOPD and AECOPD from healthy subjects. Because the level of miRNA-101-3p.1 was significantly higher in SCOPD patients and AECOPD patients than that in healthy patients, the ROC curve revealed high AUC values. Furthermore, similar AUC values in SCOPD patients and AECOPD patients may be affected by the fewer number of COPD patients enrolled and differences in severity of COPD. However, the analysis did not 
have satisfactory accuracy to discriminate between SCOPD and AECOPD due to the influence of COPD patients with GOLD III or GOLD IV. This finding is largely consistent with a previous study in which biomarkers were unable to discriminate AECOPD from COPD (29,30). At present, the clinical diagnosis of SCOPD and AECOPD is still mainly dependent on comprehensive analysis of clinical symptoms, laboratory assay and imaging examinations, which is susceptible to the subjective judgment of doctors and the behavioral performance of patients (31,32). miRNA-101-3p.1 as an indicator is helpful in judging disease severity, patient susceptibility, disease status, and disease progression, all of which are clinically significant for guiding the rational use of drugs, prognostic evaluation and predicting treatment response.

Chronic airway inflammation is an important factor contributing to airway remodeling and progressive airway obstruction in COPD patients (33). Clinical and pathological studies indicated that overexpression of inflammatory cytokines can cause histopathological injury and inhibit the proliferation of airway cells, causing airway reconstruction and pulmonary function decline $(34,35)$. The present results demonstrated that increasing miRNA-101-3p.1 promoted PBMC proliferation, a finding largely consistent with a study by Kim et al (36) in which miR-101-3p played an important role in promoting proliferation and inhibiting endothelial cell apoptosis. Increased PBMC proliferation promotes the infiltration of large numbers of mononuclear cells in the lung. Mononuclear cells then differentiate into macrophages, leading to a continuous deterioration of inflammatory response. Several recent studies have revealed that upregulation of IL-13, IL-9, IL-1, and TNF- $\alpha$ induces inflammatory cell infiltration, including macrophages, neutrophils and dendritic cells that contribute to the airway remodeling and destruction of lung tissue in COPD (37-39). Furthermore, Zhang et al (40) also revealed that HIF-1 $\alpha$ overexpression aggravated COPD pathological changes by upregulating the level IL-13, IL-9, IL-1, and TNF- $\alpha$. In the present study, it was also determined that increasing miRNA-101-3p.1 induced the expression of inflammatory cytokines, including IL-13, IL-9, IL-1 and TNF- $\alpha$, indicating that miRNA-101-3p.1 may be involved in pulmonary inflammation. Systemic inflammation is also considered to be closely associated with increased mortality of COPD patients. Therefore, such observations further support the conclusion that miRNA-101-3p.1 participates in the development of COPD.

The functions of miRNAs are considered to strongly depend on post-transcriptional regulation of target proteins (41). In the present study, bioinformatics analysis and functional verification indicated that miRNA-101-3p.1 directly inhibited pVHL and UBE2D1 expression by binding to their 3'UTR. Several recent studies demonstrated that pVHL and Cul2 can form a complex and cooperate with E2 ubiquitin-binding enzyme to induce the ubiquitination and degradation of HIF-1 $\alpha(42,43)$. In addition, HIF-1 $\alpha$ can activate the transcription of oxygen-sensitive genes to maintain survival under hypoxic conditions, and COPD is considered to be a chronic hypoxic lung disease (44-46). Therefore, the relationship between miRNA-101-3p.1 and HIF-1 $\alpha$ was explored. It was revealed that HIF- $1 \alpha$ expression significantly increased with ectopic expression of miRNA-101-3p.1 or decreasing expression of pVHL and UBE2D1, indicating that miRNA-101-3p.1 aggravates COPD in a HIF-1 $\alpha$-dependent manner. This also explains why miRNA-101-3p.1 has high resolution for COPD diagnosis. However, the regulatory mechanism in which UBE2D1 downregulates the HIF-1 $\alpha$ level is still unclear. UBE2D1 may participate in pVHL-mediated HIF-1 $\alpha$ degradation as an E2 ubiquitin-binding enzyme. Subsequently, the effect of HIF-1 $\alpha$ on the EGFR/PI3K/AKT signaling pathway, an inflammatory-related signaling pathway, was investigated. Notably, HIF-1 $\alpha$ overexpression was propitious to the upregulation of p-PI3K/PI3K, p-AKT/AKT, p-IкB $\alpha / \mathrm{I} \kappa \mathrm{B} \alpha$, EGFR and $\mathrm{NF}-\mathrm{KB}$, demonstrating the activation of the EGFR/PI3K/AKT signaling pathway. In agreement with previous findings, HIF-1 $\alpha$ upregulated the expression of inflammatory factors by activating the EGFR/PI3K/AKT pathway (40). Collectively, the present study revealed that increasing miRNA-101-3p.1 aggravates COPD through HIF-1 $\alpha$-dependent activation of the EGFR/PI3K/AKT signaling pathway.

In summary, the value of miRNA-101-3p.1 for diagnosis of SCOPD and AECOPD was determined, and the molecular mechanism by which miRNA-101-3p.1 facilitates COPD progression was explored. The results revealed that the level of miRNA-101-3p.1 in PBMCs of COPD patients was significantly increased, especially in AECOPD patients and was significantly correlated with the increase of CAT score and deterioration of pulmonary function. Furthermore, by binding to their 3'UTRs, miRNA-101-3p.1 directly inhibited pVHL and UBE2D1 expression and co-upregulated HIF-1 $\alpha$, which activated the EGFR/PI3K/AKT signaling pathway. The ability of miRNA-101-3p.1 to differentiate COPD will allow for more accurate diagnosis of individual patients, complementing standard clinical techniques.

\section{Acknowledgements}

Not applicable.

\section{Funding}

The present research was supported by Hospital Research Fund (B1519) and Clinical Research Fund of Zhejiang Medical Association (2016ZYC-A20).

\section{Availability of data and materials}

All data generated or analyzed during this study are included in this published article.

\section{Authors' contributions}

SC, ZZ and LC designed the study, and wrote and revised the manuscript. SC and JZ performed the experiments and analyzed the data. All authors read and approved the final manuscript and agree to be accountable for all aspects of the work in ensuring that questions related to the accuracy or integrity of any part of the work are appropriately investigated and resolved.

\section{Ethics approval and consent to participate}

All study protocols were approved by The Ethics and Scientific Committees of Zhejiang University. Before study participation, written informed consent was provided by each participant 


\section{Patient consent for publication}

Not applicable.

\section{Competing interests}

The authors declare that they have no competing interests.

\section{References}

1. Marçôa R, Rodrigues DM, Dias M, Ladeira I, Vaz AP, Lima R and Guimarães M: Classification of chronic obstructive pulmonary disease (COPD) according to the new global initiative for chronic obstructive lung disease (GOLD) 2017: Comparison with GOLD 2011. COPD 15: 21-26, 2018.

2. McCullagh BN, Comellas AP, Ballas ZK, Newell JD Jr, Zimmerman MB and Azar AE: Antibody deficiency in patients with frequent exacerbations of chronic obstructive pulmonary disease (COPD). PLoS One 12: e0172437, 2017.

3. Wang Z, Wang C and Yang X: Efficacy of salmeterol and formoterol combination treatment in mice with chronic obstructive pulmonary disease. Mol Med Rep 15: 1538-1545, 2018.

4. Mohammed J, Derom E, Van Oosterwijck J, Da Silva H and Calders P: Evidence for aerobic exercise training on the autonomic function in patients with chronic obstructive pulmonary disease (COPD): A systematic review. Physiotherapy 104: 36-45, 2018.

5. Engel M,Endesfelder D, Schloter-Hai B, Kublik S, Granitsiotis MS Boschetto P, Stendardo M, Barta I, Dome B, Deleuze JF, et al: Influence of lung $\mathrm{CT}$ changes in chronic obstructive pulmonary disease (COPD) on the human lung microbiome. PLoS One 12: e0180859, 2017.

6. Khan DM, Ullah A, Randhawa FA, Iqtadar S, Butt NF and Waheed K: Role of vitamin D in reducing number of acute exacerbations in chronic obstructive pulmonary disease (COPD) patients. Pak J Med Sci 33: 610-4, 2017.

7. Allers M, Langejuergen J, Gaida A, Holz O, Schuchardt S, Hohlfeld JM and Zimmermann S: Measurement of exhaled volatile organic compounds from patients with chronic obstructive pulmonary disease (COPD) using closed gas loop GC-IMS and GC-APCI-MS. J Breath Res 10: 026004, 2016.

8. Spero K, Khorfan F and Bayasi G: The over diagnosis of COPD in hospitalized patients. Chest 150: 921A, 2016.

9. Yang IA, Brown JL, George J, Jenkins S, McDonald CF McDonald VM, Phillips K, Smith BJ, Zwar NA and Dabscheck E: COPD-X Australian and New Zealand guidelines for the diagnosis and management of chronic obstructive pulmonary disease: 2017 update. Med J Aust 207: 436-442, 2017.

10. Peat R, Furlong J, Byrne T, Young R, Kangombe A, Elkin T, Renwick S, Russell D, Oelbaum S, Burhan H and Walke PP: P198 Anchoring copd screening to drug services in heroin and crack smokers to improve diagnosis. Thorax 71: A192.1-A192, 2016

11. Wang Q, Yu H, Yu H, Ma M, Ma YL and Li R: miR-223-3p/TIAL1 interaction is involved in the mechanisms associated with the neuroprotective effects of dexmedetomidine on hippocampal neuronal cells in vitro. Mol Med Rep 19: 805-812, 2019.

12. Jin R, Hu S, Liu X, Guan R, Lu L and Lin R: Intranasal instillation of miR-410 targeting IL-4/IL-13 attenuates airway inflammation in OVA-induced asthmatic mice. Mol Med Rep 19 895-900, 2019.

13. Shen W, Liu J, Zhao G, Fan M, Song G, Zhang Y, Weng Z and Zhang Y: Repression of Toll-like receptor-4 by microRNA-149-3p is associated with smoking-related COPD. Int J Chron Obstruct Pulmon Dis 12: 705-15, 2017.

14. Liu X, Qu J, Xue W, He L, Wang J, Xi X, Liu X, Yin Y and Qu Y: Bioinformatics-based identification of potential microRNA biomarkers in frequent and non-frequent exacerbators of COPD. Int J Chron Obstruct Pulmon Dis 13: 1217-28, 2018.

15. Sheng Y, Li J, Zou C, Wang S, Cao Y, Zhang J, Huang A and Tang H: Downregulation of miR-101-3p by hepatitis B virus promotes proliferation and migration of hepatocellular carcinoma cells by targeting Rab5a. Arch Virol 159: 2397-2410, 2014.

16. Hassan F, Nuovo GJ, Crawford M, Boyaka PN, Kirkby S, Nana-Sinkam SP and Cormet-Boyaka E: MiR-101 and miR-144 regulate the expression of the CFTR chloride channel in the lung. PLoS One 7: e50837, 2012.
17. Livak KJ and Schmittgen TD: Analysis of relative gene expression data using real-time quantitative PCR and the 2(-Delta Delta C(T)) method. Methods 25: 402-408, 2001

18. Arbillaga-Etxarri A, Gimeno-Santos E, Barberan-Garcia A, Benet M, Borrell E, Dadvand P, Foraster M, Marín A, Monteagudo M, Rodriguez-Roisin R, et al: Socio-environmental correlates of physical activity in patients with chronic obstructive pulmonary disease (COPD). Thorax 72: 796-802, 2017.

19. Hoogendoorn M, Feenstra TL, Asukai Y, Briggs AH, Hansen RN, Leidl R, Risebrough N, Samyshkin Y, Wacker M and Rutten-van Mölken MP: External validation of health economic decision models for chronic obstructive pulmonary disease (COPD): Report of the third COPD modeling meeting. Value Health 20: 397-403,2017.

20. Zhao E, Maj T, Kryczek I, Wei L, Ke W, Zhao L, Wei S, Crespo J, Wan S, Vatan L, et al: Cancer mediates effector T cell dysfunction by targeting microRNAs and $\mathrm{EZH} 2$ via glycolysis restriction. Nat Immunol 17: 95-103, 2016.

21. Pichiorri F, Suh SS, Rocci A, De Luca L, Taccioli C, Santhanam R, Zhou W, Benson DM Jr, Hofmainster C, Alder $\mathrm{H}$, et al: Downregulation of p53-inducible microRNAs 192, 194, and 215 impairs the p53/MDM2 autoregulatory loop in multiple myeloma development. Cancer Cell 18: 367-381, 2010.

22. O'Leary L, Sevinç K, Papazoglou IM, Tildy B, Detillieux K, Halayko AJ, Chung KF and Perry MM: Airway smooth muscle inflammation is regulated by microRNA-145 in COPD. FEBS Lett 590: 1324-1334, 2016.

23. Xu Y, Yao Y, Jiang X, Zhong X, Wang Z, Li C, Kang P, Leng K, Ji D, Li Z, et al: SP1-induced upregulation of lncRNA SPRY4-IT1 exerts oncogenic properties by scaffolding EZH2/LSD1/DNMT1 and sponging miR-101-3p in cholangiocarcinoma. J Exp Clin Canc Res 37: 81, 2018.

24. Liang H, Yu T, Han Y, Jiang H, Wang C, You T, Zhao X, Shan H, Yang R, Yang L, et al: LncRNA PTAR promotes EMT and invasion-metastasis in serous ovarian cancer by competitively binding miR-101-3p to regulate ZEB1 expression. Mol Cancer 17: 119, 2018

25. Hung $\mathrm{CH}, \mathrm{Hu} \mathrm{TH}$, Lu SN, Kuo FY, Chen $\mathrm{CH}$, Wang JH, Huang CM, Lee CM, Lin CY, Yen YH and Chiu YC: Circulating microRNAs as biomarkers for diagnosis of early hepatocellular carcinoma associated with hepatitis B virus. Int J Cancer 138 : 714-720, 2016.

26. Karakas M, Schulte C, Appelbaum S, Ojeda F, Lackner KJ, Münzel T, Schnabel RB, Blankenberg S and Zeller T: Circulating microRNAs strongly predict cardiovascular death in patients with coronary artery disease-results from the large AtheroGene study. Eur Heart J 38: 516-523, 2017.

27. Ramshankar V and Krishnamurthy A: Lung cancer detection by screening-presenting circulating miRNAs as a promising next generation biomarker breakthrough. Asian Pac J Cancer Prev 14: 2167-2172, 2013

28. Su H, Yang JR, Xu T, Huang J, Xu L, Yuan Y and Zhuang SM: MicroRNA-101, down-regulated in hepatocellular carcinoma, promotes apoptosis and suppresses tumorigenicity. Cancer Res 69: 1135-1142, 2009.

29. Chen YR, Chen V, Hollander Z, Leipsic JA, Hague CJ, Demarco ML, FitzGerald JM, McManus BM, Ng RT and Sin DD: $\mathrm{C}$-reactive protein and $\mathrm{N}$-terminal prohormone brain natriuretic peptide as biomarkers in acute exacerbations of COPD leading to hospitalizations. PLoS One 12: e0174063, 2017.

30. Song Y, Chen R, Zhan Q, Chen S, Luo Z, Ou J and Wang C: The optimum timing to wean invasive ventilation for patients with AECOPD or COPD with pulmonary infection. Int J Chron Obstruct Pulmon Dis 11: 535-542, 2016.

31. Mouronte-Roibás C, Leiro-Fernández V, Ruano-Raviña A, Ramos-Hernández C, Abal-Arca J4, Parente-Lamelas I, Botana-Rial M, Priegue-Carrera A and Fernández-Villar A: Chronic obstructive pulmonary disease in lung cancer patients: Prevalence, underdiagnosis, and clinical characterization. Respiration 95: 414-421, 2018.

32. Cosio BG, Soriano JB, López-Campos JL, Calle-Rubio M, Soler-Cataluna JJ, de-Torres JP, Marín JM, Martínez-Gonzalez C, de Lucas P, Mir I, et al: Defining the Asthma-COPD overlap syndrome in a COPD cohort. Chest 149: 45-52, 2016.

33. Green CE and Turner AM: The role of the endothelium in asthma and chronic obstructive pulmonary disease (COPD). Resp Res 18: 20, 2017.

34. Leiro-Fernández V, Priegue Carrera A and Fernández-Villar A: Efficacy of double bronchodilation (LABA+LAMA) in patients with chronic obstructive pulmonary disease (COPD) and lung cancer. Arch Bronconeumol 52: 622-623, 2016 (In English, Spanish). 
35. Kokturk N, Baha A, Oh YM, Young Ju J and Jones PW: Vitamin D deficiency: What does it mean for chronic obstructive pulmonary disease (COPD)? a compherensive review for pulmonologists. Clin Respir J 12: 382-397, 2016.

36. Kim JH, Lee DK, Kim J, Choi S, Park W, Ha KS, Kim TH, Choe J, Won MH, Kwon YG and Kim YM: A miRNA-101-3p/Bim axis as a determinant of serum deprivation-induced endothelial cel apoptosis. Cell Death Dis 8: e2808, 2017.

37. Zou SC, Pang LL, Mao QS, Wu SY and Xiao QF: IL-9 exacerbates the development of chronic obstructive pulmonary disease through oxidative stress. Eur Rev Med Pharmacol Sci 22: 8877-8884, 2018.

38. Kang MJ, Choi LM, Kim BH, Lee CM, Cho WK, Choe G, Kim DH, Lee CG and Elias JA: IL-18 induces emphysema and airway and vascular remodeling via IFN- $\gamma$, IL-17A, and IL-13. Am J Respir Crit Care Med 11: 1205-1217, 2012.

39. Wang Y, Xu J, Meng Y, Adcock IM and Yao X: Role of inflammatory cells in airway remodeling in COPD. Int J Chron Obstruct Pulmon Dis 13: 3341-3348, 2018.

40. Zhang HX, Yang JJ, Zhang SA, Zhang SM, Wang JX, Xu ZY and Lin RY: HIF-1 $\alpha$ promotes inflammatory response of chronic obstructive pulmonary disease by activating EGFR/PI3K/AKT pathway. Eur Rev Med Pharmacol Sci 22: 6077-6084, 2018.
41. Wang J, Liang $\mathrm{H}, \mathrm{Ge} \mathrm{H}$, Guo XL, Gu D and Yuan Y: MicroRNA-363-3p inhibits hepatocarcinogenesis by targeting HMGA2 and is associated with liver cancer stage. Mol Med Rep 19: 935-942, 2019.

42. Min JH, Yang H, Ivan M, Gertler F, Kaelin WG Jr and Pavletich NP: Structure of an HIF-1alpha-pVHL complex: Hydroxyproline recognition in signaling. Science 296: 1886-1889, 2002.

43. Miller F, Kentsis A, Osman R and Pan ZQ: Inactivation of VHL by tumorigenic mutations that disrupt dynamic coupling of the pVHL.hypoxia-inducible transcription factor-1alpha complex. J Biol Chem 280: 7985-7996, 2005.

44. Semba H, Takeda N, Isagawa T, Sugiura Y, Honda K, Wake M, Miyazawa H, Yamaguchi Y, Miura M, Jenkins DM, et al: HIF-1 $\alpha$-PDK1 axis-induced active glycolysis plays an essential role in macrophage migratory capacity. Nat Commun 7: 11635, 2016.

45. Shih JW, Chiang WF, Wu ATH, Wu MH, Wang LY, Yu YL, Hung YW, Wang WC, Chu CY, Hung CL, et al: Long noncoding RNA LncHIFCAR/MIR31HG is a HIF-1 $\alpha$ co-activator driving oral cancer progression. Nat Commun 8: 15874, 2017.

46. Sundh J and Ekström M: Risk factors for developing hypoxic respiratory failure in COPD. Int $\mathrm{J}$ Chron Obstruct Pulmon Dis 12: 2095-2100, 2017. 